\title{
Metric 1-median selection with fewer queries*
}

\author{
Ching-Lueh Chang ${ }^{\dagger}$
}

August 23, 2021

\begin{abstract}
Let $h: \mathbb{Z}^{+} \rightarrow \mathbb{Z}^{+} \backslash\{1\}$ be any function such that $h(n)$ and $\left\lceil n^{1 / h(n)}\right\rceil$ are computable from $n$ in $O\left(h(n) \cdot n^{1+1 / h(n)}\right)$ time. We show that given any $n$ point metric space $(M, d)$, the problem of finding $\operatorname{argmin}_{i \in M} \sum_{j \in M} d(i, j)$ (breaking ties arbitrarily) has a deterministic, $O\left(h(n) \cdot n^{1+1 / h(n)}\right)$-time, $O\left(n^{1+1 / h(n)}\right)$-query, $(2 h(n))$-approximation and nonadaptive algorithm. Our proofs modify those of Chang [3, 4] with the following improvements:
\end{abstract}

- We improve Chang's [3] query complexity of $O\left(h(n) \cdot n^{1+1 / h(n)}\right)$ to $O\left(n^{1+1 / h(n)}\right)$, everything else being equal.

- Chang's [4] unpublished work establishes our result only when $n$ is a perfect $(h(n))$ th power.

\section{Introduction}

A metric space is a nonempty set $M$ endowed with a function $d: M \times M \rightarrow[0, \infty)$ such that

$$
\begin{aligned}
d(x, x) & =0, \\
d(x, y) & >0, \\
d(x, y) & =d(y, x), \\
d(x, y)+d(y, z) & \geq d(x, z)
\end{aligned}
$$

for all distinct $x, y, z \in M$. Given an $n$-point metric space $(M, d)$, METRIC 1-MEDIAN asks for $\operatorname{argmin}_{i \in M} \sum_{j \in M} d(i, j)$, breaking ties arbitrarily. As usual, an algorithm for METRIC 1-MEDIAN may make any query $(x, y) \in M^{2}$ to obtain $d(x, y)$ in $O(1)$ time. By convention, if its sequence of queries depends only on

*A preliminary version of this paper appears in Proceedings of the 2017 IEEE International Conference on Applied System Innovation, Sapporo, Japan.

$\dagger$ Department of Computer Science and Engineering, Yuan Ze University, Taoyuan, Taiwan. Email: clchang@saturn.yzu.edu.tw 
$M$ but not on $d$, then it is said to be nonadaptive. For all $c \geq 1$, a $c$-approximate 1-median of $(M, d)$ refers to a point $z \in M$ satisfying

$$
\sum_{x \in M} d(z, x) \leq c \cdot \min _{y \in M} \sum_{x \in M} d(y, x) .
$$

A $c$-approximation algorithm for METRIC 1-MEDIAN is one that always outputs a $c$-approximate 1-median. As usual for metric-space problems, we adopt the real RAM model. In particular, we need $O(1)$-time real-number additions, multiplications and comparisons.

Metric 1-Median has a Monte-Carlo $O\left(n / \epsilon^{2}\right)$-time $(1+\epsilon)$-approximation algorithm for all $\epsilon>0[9,10]$. Kumar et al. [13] give a Monte-Carlo $O(D$. $\left.\exp \left(1 / \epsilon^{O(1)}\right)\right)$-time $(1+\epsilon)$-approximation algorithm for METRIC 1-MEDIAN in $\mathbb{R}^{D}$, where $\epsilon>0$ and $D \in \mathbb{Z}^{+}$. Algorithms abound for the more general metric $k$-median problem $[6,8,13]$. In social network analysis, the closeness centrality of an actor is the reciprocal of the average distance from that actor to others [15]. It is a popular measure of importance [15]. So METRIC 1-MEDIAN can be interpreted as finding one of the most important actors. Furthermore, METRIC 1-MEDIAN generalizes the classical median selection [7].

This paper focuses on deterministic sublinear-time algorithms for METRIC 1-MEDIAN, where "sublinear" means " $o\left(n^{2}\right)$ " by convention because there are $n(n-1) / 2$ nonzero distances [9]. In particular, we shall improve the following theorem:

Theorem 1 (Implicit in $[3,16]$ ). Let $h: \mathbb{Z}^{+} \rightarrow \mathbb{Z}^{+} \backslash\{1\}$ be any function such that $h(n)$ and $\left\lceil n^{1 / h(n)}\right\rceil$ are computable from $n$ in $O\left(h(n) \cdot n^{1+1 / h(n)}\right)$ time. Then METRIC 1-MEDIAN has a deterministic, $O\left(h(n) \cdot n^{1+1 / h(n)}\right)$-time, $O\left(h(n) \cdot n^{1+1 / h(n)}\right)$ query, $(2 h(n))$-approximation and nonadaptive algorithm.

Theorem 1 is rather general: Together with Chang's lower bound, it implies $\min \left\{c \geq 1 \mid\right.$ METRIC 1-MEDIAN has a deterministic $O\left(n^{1+\epsilon}\right)$-query $c$-approx. algorithm $\}$ $=\min \left\{c \geq 1 \mid\right.$ METRIC 1-MEDIAN has a deterministic $O\left(n^{1+\epsilon}\right)$-time $c$-approx. algorithm $\}$$$
=2\left\lceil\frac{1}{\epsilon}\right\rceil
$$

for all constants $\epsilon \in(0,1)[5]$.

To prove Theorem 1, Chang [3] designs a function $\tilde{d}:\{0,1, \ldots, n-1\}^{2} \rightarrow$ $[0, \infty)$ such that a 1 -median w.r.t. $\tilde{d}$ is $(2 h(n))$-approximate w.r.t. $d$ and is computable in $O\left(h(n) \cdot n^{1+1 / h(n)}\right)$ time. However, $\tilde{d}(\cdot, \cdot)$ depends on $\Theta\left(h(n) \cdot n^{1+1 / h(n)}\right)$ distances of $d$, forbidding us to improve the query complexity of $O\left(h(n) \cdot n^{1+1 / h(n)}\right)$ in Theorem 1. Wu's [16] algorithm also makes $\Theta\left(h(n) \cdot n^{1+1 / h(n)}\right)$ queries. In contrast, we design a new function, $\hat{d}$, that depends on only $\Theta\left(n^{1+1 / h(n)}\right)$ distances of $d$ and is otherwise similar to Chang's $\tilde{d}$. This results in a deterministic, $O\left(h(n) \cdot n^{1+1 / h(n)}\right)$-time, $O\left(n^{1+1 / h(n)}\right)$-query, $(2 h(n))$-approximation and nonadaptive algorithm for METRIC 1-MEDIAN, shaving the factor of $h(n)$ from the 
query complexity in Theorem 1. So our result strengthens Theorem 1 whenever $h(n)=\omega(1) .{ }^{1}$ The idea of our construction of $\hat{d}$ comes from an unpublished paper of Chang [4]. Aside from the design of $\hat{d}$, our proofs modify those of Chang [3] by cumbersome brute force.

As a corollary to our result, METRIC 1-MEDIAN has a deterministic, $O(\exp (O(1 / \epsilon))$. $n \log n)$-time, $O(\exp (O(1 / \epsilon)) \cdot n)$-query, $(\epsilon \log n)$-approximation and nonadaptive algorithm for all constants $\epsilon>0$. For each constant $D \geq 1$, METRIC 1-MEDIAN in $\mathbb{R}^{D}$ has a deterministic, $O(k(\epsilon) \cdot n \log n)$-time, $O(k(\epsilon) \cdot n)$-space and $(1+\epsilon)$ approximation algorithm for some function $k:(0, \infty) \rightarrow(0, \infty)$ [2, Theorem 9]. But for general metrics, we do not even know whether deterministic $O(n \log n)$ time algorithms can be $o(\log n)$-approximate.

Our improvement of the query complexity in Theorem 1 to $O\left(n^{1+1 / h(n)}\right)$ almost matches the following lower bound, leaving a multiplicative gap of about $h(n)$ on the query complexity:

Theorem 2 ([5]). METRIC 1-MEDIAN has no deterministic o $\left(n^{1+1 /(h(n)-1)} / h(n)\right)$ query $(2 h(n) \cdot(1-\epsilon))$-approximation algorithms for any constant $\epsilon>0$ and any $h: \mathbb{Z}^{+} \rightarrow \mathbb{Z}^{+} \backslash\{1\}$ satisfying $h=o\left(n^{1 /(h(n)-1)}\right)$.

Although all deterministic algorithms for METRIC 1-MEDIAN are provably outperformed by Indyk's randomized algorithm [5], their limits are nonetheless worth studying because randomness is a computational resource from the viewpoint of theoretical computer science. For example, after oblivious permutation routing on the $n$-node hypercube is known to have a randomized $O(\log n)$-time algorithm [14], its deterministic time complexity of $\Omega(\sqrt{n} / \log n)$ is still considered interesting [12]. Similarly, the deterministic time complexities of primality testing and polynomial identity testing are considered important (and the latter still open) even after their randomized counterparts are known to be efficient [1, 11].

Before diving into details, we give an intuitive exposition as to why we have an $O\left(n^{1+1 / h(n)}\right)$-query $(2 h(n))$-approximation algorithm.

An intuitive exposition. Take $h \equiv 3$ for an example. We now intuitively explain the approximation ratio of $2 h=6$. For simplicity, assume $n$ to be cubic and let $t=n^{1 / 3}$. Clearly,

$$
\{0,1, \ldots, n-1\}=\left\{s_{2} t^{2}+s_{1} t+s_{0} \mid s_{2}, s_{1}, s_{0} \in\{0,1, \ldots, t-1\}\right\} .
$$

For all $i \in\{0,1, \ldots, n-1\}$ and $s_{2}, s_{1}, s_{0} \in\{0,1, \ldots, t-1\}$,

$$
\begin{aligned}
& \hat{d}\left(i, i t^{3}+s_{2} t^{2}+s_{1} t+s_{0} \bmod n\right) \\
\equiv & d\left(i, i t+s_{2} \bmod n\right) \\
+ & d\left(i t+s_{2} \bmod n, i t^{2}+s_{2} t+s_{1} \bmod n\right) \\
+ & d\left(i t^{2}+s_{2} t+s_{1} \bmod n, i t^{3}+s_{2} t^{2}+s_{1} t+s_{0} \bmod n\right) .
\end{aligned}
$$

\footnotetext{
${ }^{1}$ For a concrete example, if we look for $\tilde{O}(n)$-time algorithms, then we will need $h(n)=\omega(1)$ in Theorem 1.
} 
By Eq. (1), the domain of $\hat{d}$ is $\{0,1, \ldots, n-1\}^{2}$.

Let $\boldsymbol{u}$ and $\boldsymbol{v}$ be independent and uniformly random elements of $\{0,1, \ldots, n-$ $1\}$. Assuming $\operatorname{gcd}(t, n)=1$ for now, it is nontrivial but provable that

$$
\hat{d}(\boldsymbol{u}, \boldsymbol{v})=d\left(\boldsymbol{u}, \boldsymbol{c}_{1}\right)+d\left(\boldsymbol{c}_{1}, \boldsymbol{c}_{2}\right)+d\left(\boldsymbol{c}_{2}, \boldsymbol{v}\right)
$$

for uniformly random (but dependent) elements $\boldsymbol{c}_{1}$ and $\boldsymbol{c}_{2}$ of $\{0,1, \ldots, n-1\}$. So for a 1-median OPT w.r.t. d, the triangle inequality implies

$$
\begin{aligned}
\hat{d}(\boldsymbol{u}, \boldsymbol{v}) & \leq d(\mathrm{OPT}, \boldsymbol{u})+d\left(\mathrm{OPT}, \boldsymbol{c}_{1}\right) \\
& +d\left(\mathrm{OPT}, \boldsymbol{c}_{1}\right)+d\left(\mathrm{OPT}, \boldsymbol{c}_{2}\right) \\
& +d\left(\mathrm{OPT}, \boldsymbol{c}_{2}\right)+d(\mathrm{OPT}, \boldsymbol{v})
\end{aligned}
$$

whose right-hand side sums 6 distances from OPT to uniformly random points. Now take expectations on both sides to see that the average $\hat{d}$-distance is at most 6 times the average d-distance from OPT to all points. This hints that a 1-median w.r.t. $\hat{d}$ is a 6-approximate 1-median w.r.t. $d$.

To find a 1-median w.r.t. $\hat{d}$, we shall find $\sum_{j=0}^{n-1} \hat{d}(i, j)$ for all $i \in\{0,1, \ldots, n-$ 1\}. By Eq. (1),

$$
\sum_{j=0}^{n-1} \hat{d}(i, j)=\sum_{s_{2}, s_{1}, s_{0}=0}^{t-1} \hat{d}\left(i, i t^{3}+s_{2} t^{2}+s_{1} t+s_{0} \bmod n\right) .
$$

Furthermore, by Eq. (2),

$$
\begin{aligned}
& \sum_{s_{2}, s_{1}, s_{0}=0}^{t-1} \hat{d}\left(i, i t^{3}+s_{2} t^{2}+s_{1} t+s_{0} \bmod n\right) \\
= & \sum_{s_{2}, s_{1}, s_{0}=0}^{t-1}\left[d\left(i, i t+s_{2} \bmod n\right)\right. \\
+ & d\left(i t+s_{2} \bmod n, i t^{2}+s_{2} t+s_{1} \bmod n\right) \\
& \left.+d\left(i t^{2}+s_{2} t+s_{1} \bmod n, i t^{3}+s_{2} t^{2}+s_{1} t+s_{0} \bmod n\right)\right] .
\end{aligned}
$$

Albeit nontrivial, the right-hand side of Eq. (3) is a 3-level sum (so called because it is taken over three variables $s_{2}, s_{1}$ and $s_{0}$ ) expressible using 2-level sums of a similar form. So, unsurprisingly, it can be found by dynamic programming (that builds up sums with increasing levels).

Now comes the key question: How do we shave the factor of h from the query complexity in Theorem 1? Observe that $\hat{d}$ in Eq. (2) depends only on distances of the form $d(j, j t+s \bmod n)$, where $j \in\{0,1, \ldots, n-1\}$ and $s \in\{0,1, \ldots, t-1\}$, for a total of nt distances. Instead, Chang's [3] $\tilde{d}$ is

$$
\begin{aligned}
& \tilde{d}\left(i, i+s_{2} t^{2}+s_{1} t+s_{0} \bmod n\right) \\
\equiv & d\left(i, i+s_{2} t^{2} \bmod n\right) \\
+ & d\left(i+s_{2} t^{2} \bmod n, i+s_{2} t^{2}+s_{1} t \bmod n\right) \\
+ & d\left(i+s_{2} t^{2}+s_{1} t \bmod n, i+s_{2} t^{2}+s_{1} t+s_{0} \bmod n\right)
\end{aligned}
$$


for all $i \in\{0,1, \ldots, n-1\}$ and $s_{2}, s_{1}, s_{0} \in\{0,1, \ldots, t-1\}$. Now verify $\tilde{d}$ to depend on all distances of the form $d\left(j, j+s t^{k} \bmod n\right)$, where $j \in\{0,1, \ldots, n-1\}$, $s \in\{0,1, \ldots, t-1\}$ and $k \in\{0,1,2\}$ - there are $3 n t=$ hnt such distances. In summary, our $\hat{d}$ depends on $h$ times fewer distances than Chang's $\tilde{d}$ does!

Everything so far is equivalent to Chang's [4] unpublished work, which assumes $n$ to be cubic (or, more generally, a perfect hth power) for Eq. (1) to hold-Gladly, this assumption can be removed by slightly modifying Chang's [3] dynamic-programming approach.

We have assumed $\operatorname{gcd}(t, n)=1$, which may be false. To get around, our construction takes suitable $t=\Theta\left(n^{1 / h}\right)$ and $\sigma \in\{0,1\}$ satisfying $\operatorname{gcd}(t, n-\sigma)=1$. Allowing $\sigma$ to be 1 makes our actual $\hat{d}$ slightly different from that in Eq. (2) and complicates our proofs significantly.

\section{The new pseudo-distance function $\hat{d}$}

Let $(\{0,1, \ldots, n-1\}, d)$ be a metric space and $h: \mathbb{Z}^{+} \rightarrow \mathbb{Z}^{+} \backslash\{1\}$ be a computable function. By Bertrand's postulate, there exists a prime number $t \in\left[\left\lceil n^{1 / h(n)}\right\rceil, 2\right.$. $\left.\left\lceil n^{1 / h(n)}\right\rceil\right]$. Clearly, $\operatorname{gcd}(n-1, n)=1$. So the primality of $t$ implies the existence of $\sigma \in\{0,1\}$ such that $\operatorname{gcd}(t, n-\sigma)=1$. For convenience, $h \equiv h(n)$. For all $j \in\{0,1, \ldots, n-1\}$, write

$$
\left(s_{h-1}(j), s_{h-2}(j), \ldots, s_{0}(j)\right) \in\{0,1, \ldots, t-1\}^{h}
$$

for the unique $t$-ary representation of $j$, following Chang [3]. ${ }^{2}$ So

$$
\sum_{\ell=0}^{h-1} s_{h-1-\ell}(j) \cdot t^{h-1-\ell}=j .
$$

For any predicate $P$, let $\chi[P]=1$ if $P$ is true and $\chi[P]=0$ otherwise.

Define

$$
d^{(n-\sigma)}(x, y) \equiv d(x \bmod (n-\sigma), y \bmod (n-\sigma))
$$

for all $x, y \in \mathbb{N}$. Clearly, $d^{(n-\sigma)}$ is symmetric and obeys the triangle inequality, just like $d$. For all $i, j \in\{0,1, \ldots, n-\sigma-1\}$, define

$$
\begin{aligned}
& \hat{d}\left(i, i t^{h}+j \bmod (n-\sigma)\right) \\
\equiv & \sum_{k=0}^{h-1} d^{(n-\sigma)}\left(i t^{k}+\sum_{\ell=0}^{k-1} s_{h-1-\ell}(j) \cdot t^{k-1-\ell}, i t^{k+1}+\sum_{\ell=0}^{k} s_{h-1-\ell}(j) \cdot t^{k-\ell}\right) .
\end{aligned}
$$

\footnotetext{
${ }^{2}$ As $t \geq\left\lceil n^{1 / h}\right\rceil$, the $t$-ary representation of any of $0,1, \ldots, n-1$ has at most $h$ digits.
} 
This and the triangle inequality for $d^{(n-\sigma)}$ imply

$$
\hat{d}\left(i, i t^{h}+j \bmod (n-\sigma)\right) \geq d^{(n-\sigma)}\left(i, i t^{h}+\sum_{\ell=0}^{h-1} s_{h-1-\ell}(j) \cdot t^{h-1-\ell}\right) .
$$

3 So by Eq. (4),

$$
\hat{d}\left(i, i t^{h}+j \bmod (n-\sigma)\right) \geq d^{(n-\sigma)}\left(i, i t^{h}+j\right)
$$

for all $i, j \in\{0,1, \ldots, n-\sigma-1\}$.

Having defined $\hat{d}\left(i, i t^{h}+j \bmod (n-\sigma)\right)$ in Eq. (6) for all $i, j \in\{0,1, \ldots, n-$ $\sigma-1\}$, the domain of $\hat{d}$ is $\{0,1, \ldots, n-\sigma-1\}^{2}{ }^{4}$ Let

$$
i^{\prime}=\underset{i=0}{\operatorname{argmin}} \sum_{j=0}^{n-1} d(i, j),
$$

breaking ties arbitrarily.

An intuitive exposition. For simplicity, assume $\sigma=0$. Pick independent and uniformly random elements $\boldsymbol{u}$ and $\boldsymbol{v}$ of $\{0,1, \ldots, n-1\}$. Taking $i \leftarrow \boldsymbol{u}$ and $j \leftarrow \boldsymbol{v}$ in Eq. (6), it can be verified that each of the $h$ summands in the right-hand side of Eq. (6) is a d-distance between uniformly random points. That is,

$$
\hat{d}\left(\boldsymbol{u}, \boldsymbol{u} t^{h}+\boldsymbol{v} \bmod n\right)=d\left(\boldsymbol{u}, \boldsymbol{c}_{1}\right)+d\left(\boldsymbol{c}_{1}, \boldsymbol{c}_{2}\right)+d\left(\boldsymbol{c}_{2}, \boldsymbol{c}_{3}\right)+\cdots+d\left(\boldsymbol{c}_{h-1}, \boldsymbol{v}\right)
$$

for uniformly random (but possibly dependent) elements $\boldsymbol{c}_{1}, \boldsymbol{c}_{2}, \ldots, \boldsymbol{c}_{h-1}$ of $\{0,1, \ldots, n-1\}$. This and the triangle inequality imply

$$
\begin{aligned}
& \hat{d}\left(\boldsymbol{u}, \boldsymbol{u} t^{h}+\boldsymbol{v} \bmod n\right) \\
\leq & \left(d\left(i^{\prime}, \boldsymbol{u}\right)+d\left(i^{\prime}, \boldsymbol{c}_{1}\right)\right)+\left(d\left(i^{\prime}, \boldsymbol{c}_{1}\right)+d\left(i^{\prime}, \boldsymbol{c}_{2}\right)\right)+\left(d\left(i^{\prime}, \boldsymbol{c}_{2}\right)+d\left(i^{\prime}, \boldsymbol{c}_{3}\right)\right)+\cdots+\left(d\left(i^{\prime}, \boldsymbol{c}_{h-1}\right)+d\left(i^{\prime}, \boldsymbol{v}\right)\right),
\end{aligned}
$$

whose right-hand side sums $2 h$ distances from $i^{\prime}$ to uniformly random points. Now take expectations on both sides to see that the average $\hat{d}$-distance is at most $2 h$ times the average d-distance from $i^{\prime}$ to all points. But, as $\sigma=0, i^{\prime}$ is a 1-median w.r.t. $d$ by Eq. (8). So it is intuitive to guess that a 1-median w.r.t. $\hat{d}$ is a (2h)approximate 1-median w.r.t. d. The next lemma, whose proof embeds the above idea with technicalities, confirms this guess. ${ }^{5}$

\footnotetext{
${ }^{3}$ Note that $i t^{k}+\sum_{\ell=0}^{k-1} s_{h-1-\ell}(j) \cdot t^{k-1-\ell}=i$ when $k=0$ and $i t^{k+1}+\sum_{\ell=0}^{k} s_{h-1-\ell}(j) \cdot t^{k-\ell}=$ $i t^{h}+\sum_{\ell=0}^{h-1} s_{h-1-\ell}(j) \cdot t^{h-1-\ell}$ when $k=h-1$.

${ }^{4}$ Note that each pair in $\{0,1, \ldots, n-\sigma-1\}^{2}$ can be written as $\left(i, i t^{h}+j \bmod (n-\sigma)\right)$ for a unique pair $(i, j) \in\{0,1, \ldots, n-\sigma-1\}^{2}$.

${ }^{5}$ When $\sigma=0, \alpha$ in Eq. (9) is a 1-median w.r.t. $\hat{d}$, and the right-hand side of Eq. (11) is $2 h$ times the total $d$-distance from a 1-median w.r.t. $d$ to all points. So Lemma 3 implies that when $\sigma=0$, a 1 -median w.r.t. $\hat{d}$ is a $(2 h)$-approximate 1 -median w.r.t. $d$.
} 
Lemma 3 (cf. [3, Lemma 4]). Let

$$
\alpha=\underset{i=0}{\operatorname{argmin}}\left(\chi[\sigma=1] \cdot d(i, n-1)+\sum_{j=0}^{n-\sigma-1} \hat{d}\left(i, i t^{h}+j \bmod (n-\sigma)\right)\right),
$$

breaking ties arbitrarily. Then

$$
\begin{aligned}
& \sum_{j=0}^{n-1} d(\alpha, j) \\
\leq & \chi[\sigma=1] \cdot d(\alpha, n-1)+\sum_{j=0}^{n-\sigma-1} \hat{d}\left(\alpha, \alpha t^{h}+j \bmod (n-\sigma)\right) \\
\leq & \left.2 h \cdot\left(\underset{i=0}{\min } \sum_{j=0}^{n-\sigma-1} d(i, j)\right)-\chi[\sigma=1] \cdot\left((2 h-1) \cdot d\left(i^{\prime}, n-1\right)-\frac{1}{n-1} \cdot \sum_{j=0}^{n-2} d\left(i^{\prime}, j\right)\right) .(11)\right)
\end{aligned}
$$

Proof. Clearly,

$$
\begin{aligned}
\sum_{j=0}^{n-1} d(\alpha, j) & \stackrel{(5)}{=} \chi[\sigma=1] \cdot d(\alpha, n-1)+\sum_{j=0}^{n-\sigma-1} d^{(n-\sigma)}(\alpha, j) \\
& =\chi[\sigma=1] \cdot d(\alpha, n-1)+\sum_{j=0}^{n-\sigma-1} d^{(n-\sigma)}\left(\alpha, \alpha t^{h}+j\right)
\end{aligned}
$$

where the second equality uses Eq. (5) and the one-to-one correspondence of $j \mapsto \alpha t^{h}+j \bmod (n-\sigma)$ for $j \in\{0,1, \ldots, n-\sigma-1\}$.

Pick $\boldsymbol{u}$ from $\{0,1, \ldots, n-\sigma-1\}$ uniformly at random. Then

$$
\begin{aligned}
& \chi[\sigma=1] \cdot d(\alpha, n-1)+\sum_{j=0}^{n-\sigma-1} d^{(n-\sigma)}\left(\alpha, \alpha t^{h}+j\right) \\
\stackrel{(7)}{\leq} & \chi[\sigma=1] \cdot d(\alpha, n-1)+\sum_{j=0}^{n-\sigma-1} \hat{d}\left(\alpha, \alpha t^{h}+j \bmod (n-\sigma)\right) \\
\stackrel{(9)}{\leq} & \mathrm{E}\left[\chi[\sigma=1] \cdot d(\boldsymbol{u}, n-1)+\sum_{j=0}^{n-\sigma-1} \hat{d}\left(\boldsymbol{u}, \boldsymbol{u} t^{h}+j \bmod (n-\sigma)\right)\right] \\
\leq & \mathrm{E}\left[\chi[\sigma=1] \cdot\left(d\left(i^{\prime}, \boldsymbol{u}\right)+d\left(i^{\prime}, n-1\right)\right)+\sum_{j=0}^{n-\sigma-1} \hat{d}\left(\boldsymbol{u}, \boldsymbol{u} t^{h}+j \bmod (n-\sigma)\right)\right] \\
= & \chi[\sigma=1] \cdot\left[\left(\frac{1}{n-\sigma} \cdot \sum_{m=0}^{n-\sigma-1} d\left(i^{\prime}, m\right)\right)+d\left(i^{\prime}, n-1\right)\right]+\mathrm{E}\left[\sum_{j=0}^{n-\sigma-1} \hat{d}\left(\boldsymbol{u}, \boldsymbol{u} t^{h}+j \bmod (n-\sigma)\right)\right]
\end{aligned}
$$

where the last inequality (resp., equality) follows from the triangle inequality for 
$d$ (resp., the uniform distribution of $\boldsymbol{u}$ over $\{0,1, \ldots, n-\sigma-1\}$ ). Furthermore,

$$
\begin{aligned}
& \mathrm{E}\left[\sum_{j=0}^{n-\sigma-1} \hat{d}\left(\boldsymbol{u}, \boldsymbol{u} t^{h}+j \bmod (n-\sigma)\right)\right] \\
& \stackrel{(6)}{=} \quad \mathrm{E}\left[\sum_{j=0}^{n-\sigma-1} \sum_{k=0}^{h-1} d^{(n-\sigma)}\left(\boldsymbol{u} t^{k}+\sum_{\ell=0}^{k-1} s_{h-1-\ell}(j) \cdot t^{k-1-\ell}, \boldsymbol{u} t^{k+1}+\sum_{\ell=0}^{k} s_{h-1-\ell}(j) \cdot t^{k-\ell}\right)\right] \\
& \leq \quad \mathrm{E}\left[\sum_{j=0}^{n-\sigma-1} \sum_{k=0}^{h-1} d^{(n-\sigma)}\left(i^{\prime}, \boldsymbol{u} t^{k}+\sum_{\ell=0}^{k-1} s_{h-1-\ell}(j) \cdot t^{k-1-\ell}\right)+d^{(n-\sigma)}\left(i^{\prime}, \boldsymbol{u} t^{k+1}+\sum_{\ell=0}^{k} s_{h-1-\ell}(j) \cdot t^{k-\ell}\right)\right] \\
& \text { (5) } \underset{\&}{=} \sum_{j=0}^{(8)} \sum_{k=0}^{n-\sigma-1}\left(\mathrm{E}\left[d\left(i^{\prime}, \boldsymbol{u} t^{k}+\sum_{\ell=0}^{k-1} s_{h-1-\ell}(j) \cdot t^{k-1-\ell} \bmod (n-\sigma)\right)\right]\right. \\
& \left.+\mathrm{E}\left[d\left(i^{\prime}, \boldsymbol{u} t^{k+1}+\sum_{\ell=0}^{k} s_{h-1-\ell}(j) \cdot t^{k-\ell} \bmod (n-\sigma)\right)\right]\right),
\end{aligned}
$$

where the inequality follows from the triangle inequality for $d^{(n-\sigma)}$.

Because $\boldsymbol{u}$ distributes uniformly at random over $\{0,1, \ldots, n-\sigma-1\}$ and $\operatorname{gcd}(t, n-\sigma)=1$ by construction,

$$
\begin{gathered}
\boldsymbol{u} t^{k}+\sum_{\ell=0}^{k-1} s_{h-1-\ell}(j) \cdot t^{k-1-\ell} \bmod (n-\sigma), \\
\boldsymbol{u} t^{k+1}+\sum_{\ell=0}^{k} s_{h-1-\ell}(j) \cdot t^{k-\ell} \bmod (n-\sigma)
\end{gathered}
$$

are uniformly random elements of $\{0,1, \ldots, n-\sigma-1\}$ for any $j \in\{0,1, \ldots, n-$ $\sigma-1\}$ and $k \in\{0,1, \ldots, h-1\} .{ }^{6} 7$ Therefore,

$$
\begin{aligned}
& \sum_{j=0}^{n-\sigma-1} \sum_{k=0}^{h-1}\left(\mathrm{E}\left[d\left(i^{\prime}, \boldsymbol{u} t^{k}+\sum_{\ell=0}^{k-1} s_{h-1-\ell}(j) \cdot t^{k-1-\ell} \bmod (n-\sigma)\right)\right]\right. \\
& \left.+\mathrm{E}\left[d\left(i^{\prime}, \boldsymbol{u} t^{k+1}+\sum_{\ell=0}^{k} s_{h-1-\ell}(j) \cdot t^{k-\ell} \bmod (n-\sigma)\right)\right]\right) \\
= & \sum_{j=0}^{n-\sigma-1} \sum_{k=0}^{h-1}\left(\frac{1}{n-\sigma} \cdot \sum_{m=0}^{n-\sigma-1} d\left(i^{\prime}, m\right)+\frac{1}{n-\sigma} \cdot \sum_{m=0}^{n-\sigma-1} d\left(i^{\prime}, m\right)\right) \\
= & 2 h \sum_{m=0}^{n-\sigma-1} d\left(i^{\prime}, m\right) .
\end{aligned}
$$

\footnotetext{
${ }^{6}$ Note that $\operatorname{gcd}\left(t^{k}, n-\sigma\right)=\operatorname{gcd}\left(t^{k+1}, n-\sigma\right)=1$.

${ }^{7}$ In essence, this observation and Eqs. (14)-(15) follow the intuitive exposition before Lemma 3.
} 
Now,

$$
\begin{aligned}
& \sum_{j=0}^{n-1} d(\alpha, j) \\
\stackrel{(12)-(13)}{\leq} & \chi[\sigma=1] \cdot d(\alpha, n-1)+\sum_{j=0}^{n-\sigma-1} \hat{d}\left(\alpha, \alpha t^{h}+j \bmod (n-\sigma)\right) \\
\stackrel{(13)-(16)}{\leq} & \chi[\sigma=1] \cdot\left[\left(\frac{1}{n-\sigma} \cdot \sum_{m=0}^{n-\sigma-1} d\left(i^{\prime}, m\right)\right)+d\left(i^{\prime}, n-1\right)\right]+2 h \sum_{m=0}^{n-\sigma-1} d\left(i^{\prime}, m\right) \\
= & \chi[\sigma=1] \cdot\left[\left(\frac{1}{n-1} \cdot \sum_{m=0}^{n-2} d\left(i^{\prime}, m\right)\right)+d\left(i^{\prime}, n-1\right)\right]+2 h \sum_{m=0}^{n-\sigma-1} d\left(i^{\prime}, m\right) \\
= & \chi[\sigma=1] \cdot\left[\left(\frac{1}{n-1} \cdot \sum_{m=0}^{n-2} d\left(i^{\prime}, m\right)\right)+d\left(i^{\prime}, n-1\right)\right] \\
+ & 2 h \cdot\left(\left(\sum_{m=0}^{n-1} d\left(i^{\prime}, m\right)\right)-\chi[\sigma=1] \cdot d\left(i^{\prime}, n-1\right)\right) \\
= & 2 h \cdot\left(\sum_{m=0}^{n-1} d\left(i^{\prime}, m\right)\right)-\chi[\sigma=1] \cdot\left((2 h-1) \cdot d\left(i^{\prime}, n-1\right)-\frac{1}{n-1} \cdot \sum_{m=0}^{n-2} d\left(i^{\prime}, m\right)\right) \\
\stackrel{(8)}{=} & 2 h \cdot\left(\min _{i=0}^{n-\sigma-1} \sum_{j=0}^{n-1} d(i, j)\right)-\chi[\sigma=1] \cdot\left((2 h-1) \cdot d\left(i^{\prime}, n-1\right)-\frac{1}{n-1} \cdot \sum_{m=0}^{n-2} d\left(i^{\prime}, m\right)\right) .
\end{aligned}
$$

Eqs. (18) and (10) coincide. Furthermore, Eqs. (18)-(19) imply Eq. (11).

An intuitive exposition. When $\sigma=0$, $\alpha$ in Eq. (9) is a (2h)-approximate 1-median w.r.t. $d$ by Lemma 3. When $\sigma=1, \alpha \in\{0,1, \ldots, n-2\}$ by Eq. (9), missing $n-1$ from the choice of $\alpha$. The next lemma considers $n-1$ as well. In particular, it shows how to pick a (2h)-approximate 1-median w.r.t. d from $\{\alpha, n-1\}$. In contrast, Chang [3, 4] has no $\sigma$ and, therefore, always includes $n-1$ in picking his analogy of $\alpha$. So the next lemma is independent of his works.

Lemma 4. Let $\alpha \in\{0,1, \ldots, n-\sigma-1\}$ be as in Eq. (9), breaking ties arbitrarily. If

$$
\chi[\sigma=1] \cdot d(\alpha, n-1)+\sum_{j=0}^{n-\sigma-1} \hat{d}\left(\alpha, \alpha t^{h}+j \bmod (n-\sigma)\right)<\sum_{j=0}^{n-1} d(n-1, j),
$$

then

$$
\sum_{j=0}^{n-1} d(\alpha, j) \leq 2 h \cdot \min _{i=0}^{n-1} \sum_{j=0}^{n-1} d(i, j)
$$

Otherwise,

$$
\sum_{j=0}^{n-1} d(n-1, j) \leq 2 h \cdot \min _{i=0}^{n-1} \sum_{j=0}^{n-1} d(i, j)
$$


Proof. Clearly,

$$
\begin{aligned}
\sum_{j=0}^{n-1} d(\alpha, j) & \stackrel{(5)}{=} \chi[\sigma=1] \cdot d(\alpha, n-1)+\sum_{j=0}^{n-\sigma-1} d^{(n-\sigma)}(\alpha, j) \\
& \stackrel{(7)}{\leq} \chi[\sigma=1] \cdot d(\alpha, n-1)+\sum_{j=0}^{n-\sigma-1} \hat{d}(\alpha, j) \\
& =\chi[\sigma=1] \cdot d(\alpha, n-1)+\sum_{j=0}^{n-\sigma-1} \hat{d}\left(\alpha, \alpha t^{h}+j \bmod (n-\sigma)\right),
\end{aligned}
$$

where the last equality uses the one-to-one correspondence of $j \mapsto \alpha t^{h}+j \bmod$ $(n-\sigma)$ for $j \in\{0,1, \ldots, n-\sigma-1\} .{ }^{8}$

Next, we separate the discussion as to whether

$$
(2 h-1) \cdot d\left(i^{\prime}, n-1\right)-\frac{1}{n-1} \cdot \sum_{m=0}^{n-2} d\left(i^{\prime}, m\right) \geq 0 .
$$

Case (1): Eq. (25) is true. By Lemma 3,

$$
\sum_{j=0}^{n-1} d(\alpha, j) \leq 2 h \cdot \min _{i=0}^{n-\sigma-1} \sum_{j=0}^{n-1} d(i, j) .
$$

Subcase (i): Eq. (20) is true. By Eqs. (20) and (23)-(24),

$$
\sum_{j=0}^{n-1} d(\alpha, j)<\sum_{j=0}^{n-1} d(n-1, j) .
$$

This and Eq. (26) imply Eq. (21).

Subcase (ii): Eq. (20) is false. By Eq. (11) of Lemma 3 and Eq. (25),

$$
\begin{aligned}
& \chi[\sigma=1] \cdot d(\alpha, n-1)+\sum_{j=0}^{n-\sigma-1} \hat{d}\left(\alpha, \alpha t^{h}+j \bmod (n-\sigma)\right) \\
\leq & 2 h \cdot \min _{i=0}^{n-\sigma-1} \sum_{j=0}^{n-1} d(i, j) .
\end{aligned}
$$

This and the negation of Eq. (20) imply

$$
\sum_{j=0}^{n-1} d(n-1, j) \leq 2 h \cdot \min _{i=0}^{n-\sigma-1} \sum_{j=0}^{n-1} d(i, j) .
$$

Eq. (27) implies Eq. (22) (note that $\sum_{j=0}^{n-1} d(n-1, j$ ) does not exceed itself).

${ }^{8}$ Note that Eq. (7) holds for all $i, j \in\{0,1, \ldots, n-\sigma-1\}$, implying that $d^{(n-\sigma)}(u, v) \leq \hat{d}(u, v)$ for all $u, v \in\{0,1, \ldots, n-\sigma-1\}$. 
Case (2): Eq. (25) is false. By the triangle inequality for $d$,

$$
\begin{aligned}
\sum_{j=0}^{n-1} d(n-1, j) & \leq \sum_{j=0}^{n-1}\left(d\left(i^{\prime}, n-1\right)+d\left(i^{\prime}, j\right)\right) \\
& =n \cdot d\left(i^{\prime}, n-1\right)+\sum_{j=0}^{n-1} d\left(i^{\prime}, j\right) .
\end{aligned}
$$

Eqs. (28)-(29) and the negation of Eq. (25) imply

$$
\begin{aligned}
\sum_{j=0}^{n-1} d(n-1, j) & <n \cdot \frac{1}{2 h-1} \cdot \frac{1}{n-1} \cdot\left(\sum_{m=0}^{n-2} d\left(i^{\prime}, m\right)\right)+\sum_{j=0}^{n-1} d\left(i^{\prime}, j\right) \\
& \leq 2 \cdot \sum_{j=0}^{n-1} d\left(i^{\prime}, j\right) \\
& \stackrel{(8)}{=} 2 \cdot \min _{i=0}^{n-\sigma-1} \sum_{j=0}^{n-1} d(i, j)
\end{aligned}
$$

where the second inequality uses $h \geq 2$. Eqs. (30)-(31) imply Eq. (22).

Subcase (a): Eq. (20) is false. We have proved Eq. (22), as desired.

Subcase (b): Eq. (20) is true. By Eqs. (20) and (23)-(24),

$$
\sum_{j=0}^{n-1} d(\alpha, j)<\sum_{j=0}^{n-1} d(n-1, j) .
$$

This and Eq. (22) (which has been proved) give Eq. (21).

\section{Dynamic programming}

By Lemma 4, one of $\alpha$ and $n-1$ is a (2h)-approximate 1-median. This section finds $\alpha$ by dynamic programming. Details follow.

Define $\left(s_{h-1}^{\prime}, s_{h-2}^{\prime}, \ldots, s_{0}^{\prime}\right) \in\{0,1, \ldots, t-1\}^{h}$ to be the $t$-ary representation of $n-\sigma-1$. So $\sum_{r=0}^{h-1} s_{r}^{\prime} \cdot t^{r}=n-\sigma-1$. For $i \in\{0,1, \ldots, n-\sigma-1\}$ and 
$m \in\{0,1, \ldots, h-1\}$, define

$$
\begin{aligned}
f(i, m) \equiv & \sum_{s_{m}, s_{m-1}, \ldots, s_{0}=0}^{t-1} \chi\left[\sum_{r=0}^{m} s_{r} \cdot t^{r} \leq \sum_{r=0}^{m} s_{r}^{\prime} \cdot t^{r}\right] \\
& \cdot \sum_{k=0}^{m} d^{(n-\sigma)}\left(i t^{k}+\sum_{\ell=0}^{k-1} s_{m-\ell} \cdot t^{k-1-\ell}, i t^{k+1}+\sum_{\ell=0}^{k} s_{m-\ell} \cdot t^{k-\ell}\right), \\
g(i, m) \equiv & \sum_{s_{m}, s_{m-1}, \ldots, s_{0}=0}^{t-1} \sum_{k=0}^{m} d^{(n-\sigma)}\left(i t^{k}+\sum_{\ell=0}^{k-1} s_{m-\ell} \cdot t^{k-1-\ell}, i t^{k+1}+\sum_{\ell=0}^{k} s_{m-\ell} \cdot t^{k-\ell}\right) ;
\end{aligned}
$$

hence

$$
\begin{aligned}
& f(i, 0)=\sum_{s_{0}=0}^{s_{0}^{\prime}} d^{(n-\sigma)}\left(i, i t+s_{0}\right), \\
& g(i, 0)=\sum_{s_{0}=0}^{t-1} d^{(n-\sigma)}\left(i, i t+s_{0}\right) .
\end{aligned}
$$

Chang also defines functions similar to our $f(\cdot, \cdot)$ and $g(\cdot, \cdot)[3$, Eqs. (8)-(9)], based on his pseudo-distance function [3, Eq. (2)]. Instead, Eqs. (32)-(33) are based on $\hat{d}$ in Eq. (6).

The following lemma implies $f(i, h-1)=\sum_{j=0}^{n-\sigma-1} \hat{d}(i, j)$ for all $i \in\{0,1, \ldots, n-$ $\sigma-1\}$ :

Lemma 5 (cf. [3, Lemma 5]). For all $i \in\{0,1, \ldots, n-\sigma-1\}$,

$$
f(i, h-1)=\sum_{j=0}^{n-\sigma-1} \hat{d}\left(i, i t^{h}+j \bmod (n-\sigma)\right) .
$$

Proof. Representing each $j \in\{0,1, \ldots, n-\sigma-1\}$ in $t$-ary as $\left(s_{h-1}, s_{h-2}, \ldots, s_{0}\right)$,

$$
\begin{aligned}
& \sum_{j=0}^{n-\sigma-1} \sum_{k=0}^{h-1} d^{(n-\sigma)}\left(i t^{k}+\sum_{\ell=0}^{k-1} s_{h-1-\ell}(j) \cdot t^{k-1-\ell}, i t^{k+1}+\sum_{\ell=0}^{k} s_{h-1-\ell}(j) \cdot t^{k-\ell}\right) \\
= & \sum_{s_{h-1}, s_{h-2}, \ldots, s_{0}=0}^{t-1} \chi\left[\sum_{r=0}^{h-1} s_{r} \cdot t^{r} \leq n-\sigma-1\right] \\
& \cdot \sum_{k=0}^{h-1} d^{(n-\sigma)}\left(i t^{k}+\sum_{\ell=0}^{k-1} s_{h-1-\ell} \cdot t^{k-1-\ell}, i t^{k+1}+\sum_{\ell=0}^{k} s_{h-1-\ell} \cdot t^{k-\ell}\right) .
\end{aligned}
$$

Eqs. (6), (32) and (37) complete the proof (recall that $\sum_{r=0}^{h-1} s_{r}^{\prime} \cdot t^{r}=n-\sigma-$ 1). ${ }^{9}$

\footnotetext{
${ }^{9}$ By Eq. (6), the right-hand side of Eq. (36) coincides with the left-hand side of Eq. (37). By Eq. (32) and recalling that $\sum_{r=0}^{h-1} s_{r}^{\prime} \cdot t^{r}=n-\sigma-1$, the right-hand side of Eq. (37) is $f(i, h-1)$.
} 
When $\sigma=0$, a minimizer of $f(\cdot, h-1)$ is a $(2 h)$-approximate 1 -median w.r.t. $d$ by Lemmas 3 and 5 . So we want to calculate $f(i, h-1)$ for all $i \in\{0,1, \ldots, n-$ $\sigma-1\}$. Similar to [3], we do so by dynamic programming. For this purpose, we need the following recurrences for $g(\cdot, \cdot)$ and $f(\cdot, \cdot)$, whose very cumbersome (but naïve) proofs are in Appendices $\mathrm{A}-\mathrm{B}$, respectively:

Lemma 6 (cf. [3, Lemma 6]). For all $i \in\{0,1, \ldots, n-\sigma-1\}$ and $m \in$ $\{1,2, \ldots, h-1\}$,

$$
\begin{aligned}
g(i, m) & =t^{m} \sum_{s_{m}=0}^{t-1} d^{(n-\sigma)}\left(i, i t+s_{m}\right) \\
& +\sum_{s_{m}=0}^{t-1} g\left(i t+s_{m} \bmod (n-\sigma), m-1\right) .
\end{aligned}
$$

Lemma 7 (cf. [3, Lemma 7]). For all $i \in\{0,1, \ldots, n-\sigma-1\}$ and $m \in$ $\{1,2, \ldots, h-1\}$

$$
\begin{aligned}
f(i, m) & =\left(1+\sum_{r=0}^{m-1} s_{r}^{\prime} \cdot t^{r}\right) d^{(n-\sigma)}\left(i, i t+s_{m}^{\prime}\right) \\
& +t^{m} \sum_{s_{m}=0}^{s_{m}^{\prime}-1} d^{(n-\sigma)}\left(i, i t+s_{m}\right) \\
& +f\left(i t+s_{m}^{\prime} \bmod (n-\sigma), m-1\right) \\
& +\sum_{s_{m}=0}^{s_{m}^{\prime}-1} g\left(i t+s_{m} \bmod (n-\sigma), m-1\right) .
\end{aligned}
$$

Lemmas 6-7 express $g(\cdot, m)$ and $f(\cdot, m)$ using $g(\cdot, m-1), f(\cdot, m-1)$ and $d^{(n-\sigma)}(\cdot, \cdot)$. So we can find $f(\cdot, 0), f(\cdot, 1), \ldots, f(\cdot, h-1)$, in that order. A $(2 h)$ approximate 1-median can thus be found:

Lemma 8 (cf. [3, Lemma 8]). Approx.-Median in Fig. 1 outputs a (2h)-approximate 1-median w.r.t. d.

Proof. By Eqs. (34)-(35), lines 10-13 of Approx.-Median find $f(\cdot, 0)$ and $g(\cdot, 0)$. By Lemmas 6 and 7 , lines $14-23$ find $f(\cdot, m)$ and $g(\cdot, m)$ for an increasing $m \in$ $\{1,2, \ldots, h-1\}$. By Lemma 5 , line 24 picks $\alpha$ as in Eq. (9). Also by Lemma 5, the condition in line 25 is the same as Eq. (20). So by Lemma 4, lines 25-29 output a (2h)-approximate 1-median w.r.t. $d$.

By line $9,0 \leq s_{m}^{\prime} \leq t-1$ for all $m \in\{0,1, \ldots, h-1\}$. So all $d$-distances in lines 11-12, 16 and 18-19 are queried for in line 5 of the loops in lines 3-8. Instead, the $d$-distances in lines $24-25$ are queried for in line 7 .

Below is our main theorem. 
1: Pick any prime number $t \in\left[\left\lceil n^{1 / h}\right\rceil, 2 \cdot\left\lceil n^{1 / h}\right\rceil\right]$;

2: Pick any $\sigma \in\{0,1\}$ satisfying $\operatorname{gcd}(t, n-\sigma)=1$;

3: for $i=0,1, \ldots, n-\sigma-1$ do

4: $\quad$ for $s=0,1, \ldots, t-1$ do

5: $\quad$ Query for $d(i, i t+s \bmod (n-\sigma))$;

6: end for

7: $\quad$ Query for $d(n-1, i)$;

8: end for

9: $\left(s_{h-1}^{\prime}, s_{h-2}^{\prime}, \ldots, s_{0}^{\prime}\right) \leftarrow$ the $t$-ary representation of $n-\sigma-1$;

10: for $i=0,1, \ldots, n-\sigma-1$ do

11: $f[i][0] \leftarrow \sum_{s_{0}=0}^{s_{0}^{\prime}} d\left(i, i t+s_{0} \bmod (n-\sigma)\right)$;

12: $\quad g[i][0] \leftarrow \sum_{s_{0}=0}^{t-1} d\left(i, i t+s_{0} \bmod (n-\sigma)\right)$;

13: end for

14: for $m=1,2, \ldots, h-1$ do

15: $\quad$ for $i=0,1, \ldots, n-\sigma-1$ do

16: $\quad g[i][m] \leftarrow t^{m} \sum_{s_{m}=0}^{t-1} d\left(i, i t+s_{m} \bmod (n-\sigma)\right)$;

17: $\quad g[i][m] \leftarrow g[i][m]+\sum_{s_{m}=0}^{t-1} g\left[i t+s_{m} \bmod (n-\sigma)\right][m-1]$;

18: $\quad f[i][m] \leftarrow\left(1+\sum_{r=0}^{m-1} s_{r}^{\prime} \cdot t^{r}\right) d\left(i, i t+s_{m}^{\prime} \bmod (n-\sigma)\right)$;

19: $\quad f[i][m] \leftarrow f[i][m]+t^{m} \sum_{s_{m}=0}^{s_{m}^{\prime}-1} d\left(i, i t+s_{m} \bmod (n-\sigma)\right) ;$

20: $\quad f[i][m] \leftarrow f[i][m]+f\left[i t+s_{m}^{\prime} \bmod (n-\sigma)\right][m-1]$;

21: $\quad f[i][m] \leftarrow f[i][m]+\sum_{s_{m}=0}^{s_{m}^{\prime}-1} g\left[i t+s_{m} \bmod (n-\sigma)\right][m-1]$;

22: $\quad$ end for

23: end for

24: $\alpha \leftarrow \operatorname{argmin}_{i=0}^{n-\sigma-1}(\chi[\sigma=1] \cdot d(i, n-1)+f[i][h-1])$, breaking ties arbitrarily;

25: if $\chi[\sigma=1] \cdot d(\alpha, n-1)+f[\alpha][h-1]<\sum_{j=0}^{n-1} d(n-1, j)$ then

26: $\quad$ Output $\alpha$;

27: else

28: $\quad$ Output $n-1$;

29: end if

Figure 1: Algorithm Approx.-Median 
Theorem 9 (cf. [3, Theorem 9]). Let $h: \mathbb{Z}^{+} \rightarrow \mathbb{Z}^{+} \backslash\{1\}$ be any function such that $h(n)$ and $\left\lceil n^{1 / h(n)}\right\rceil$ are computable from $n$ in $O\left(h(n) \cdot n^{1+1 / h(n)}\right)$ time. Then METRIC 1-MEDIAN has a deterministic, $O\left(h(n) \cdot n^{1+1 / h(n)}\right)$-time, $O\left(n^{1+1 / h(n)}\right)$ query, $(2 h(n))$-approximation and nonadaptive algorithm.

Proof. By Lemma 8, Approx.-Median is (2h)-approximate. It is clearly deterministic and nonadaptive. ${ }^{10}$ By the well-known AKS primality test, line 1 of Approx.-Median takes time

$$
\left(\left\lceil n^{1 / h}\right\rceil+1\right) \cdot \log ^{O(1)}\left(O\left(\left\lceil n^{1 / h}\right\rceil\right)\right)=\tilde{O}(\sqrt{n}),
$$

where the equality uses $h \geq 2$. Because $\operatorname{gcd}(\cdot, \cdot)$ is polynomial-time computable and $t=O(n)$ by line 1 , line 2 takes $\log ^{O(1)} n$ time. ${ }^{11}$ Lines 3-8 make $O(n t)$ queries in $O(n t)$ time. As $0 \leq s_{0}^{\prime} \leq t-1$ by line 9, lines 10-13 take $O(n t)$ time. By precomputing $\left\{t^{i}\right\}_{i=0}^{h-1}$ and $\left\{\sum_{r=0}^{i} s_{r}^{\prime} \cdot t^{r}\right\}_{i=0}^{h-1}$ in $O(h)$ time, ${ }^{12}$ lines 14-23 take $O(h n t)$ time. $^{13}$ As $t=\Theta\left(n^{1 / h}\right)$ by line 1 , the overall time (resp., query) complexity of $O(h n t)$ (resp., $O(n t))$ is as desired.

Note that the condition on $h$ in Theorem 9 is mild; it holds for most commonly seen functions. For example, we have the following corollary:

Corollary 10. Metric 1-Median has a deterministic, $O(\exp (O(1 / \epsilon)) \cdot n \log n)$ time, $O(\exp (O(1 / \epsilon)) \cdot n)$-query, $(\epsilon \log n)$-approximation and nonadaptive algorithm for each constant $\epsilon>0$.

Proof. Take $h(n)=(\epsilon / 2) \lg n$ in Theorem 9 .

Corollary 10 is stronger than taking $h(n)=(\epsilon / 2) \lg n$ in Theorem 1. But it leaves open whether deterministic $O(n \log n)$-time or $O(n)$-query algorithms for METRIC 1-MEDIAN can be $o(\log n)$-approximate.

\footnotetext{
${ }^{10}$ Clearly, lines 3-8 of Approx.-Median make nonadaptive queries.

${ }^{11}$ In general, $\operatorname{gcd}(\cdot, \cdot)$ takes time polynomial in the lengths (not the values) of the binary representations of its inputs.

${ }^{12}$ Calculate $t^{i}=t \cdot t^{i-1}$ in the increasing order of $i \in\{1,2, \ldots, h-1\}$. Then calculate $\sum_{r=0}^{i} s_{r}^{\prime} \cdot t^{r}=s_{i}^{\prime} \cdot t^{i}+\sum_{r=0}^{i-1} s_{r}^{\prime} \cdot t^{r}$ in the increasing order of $i \in\{0,1, \ldots, h-1\}$. Because $t=\Theta\left(n^{1 / h}\right)$ and $\sum_{r=0}^{h-1} s_{r}^{\prime} \cdot t^{r}=n-\sigma-1$, the numbers appearing in calculating $\left\{t^{i}\right\}_{i=0}^{h-1}$ and $\left\{\sum_{r=0}^{i} s_{r}^{\prime} \cdot t^{r}\right\}_{i=0}^{h-1}$ are $O(h+\lg n)$ - and $O(\lg n)$-bit long, respectively. As $n^{1 / h}=\Theta(1)$ for all $h \geq \lg n$, the theorem is stronger with $h=\lg n$ than with $h>\lg n$ (because the desired time complexity, query complexity and approximation ratio of $O\left(h n^{1+1 / h}\right), O\left(n^{1+1 / h}\right)$ and $2 h$ are $O(h n), O(n)$ and $2 h$, respectively, for all $h \geq \lg n$, and the theorem is stronger with a smaller time complexity and a smaller approximation ratio). So we may assume $h \leq \lg n$ in the proof. Consequently, the numbers appearing in calculating $\left\{t^{i}\right\}_{i=0}^{h-1}$ and $\left\{\sum_{r=0}^{i} s_{r}^{\prime} \cdot t^{r}\right\}_{i=0}^{h-1}$ are $O(\lg n)$ bit long. That arithmetic over $O(\lg n)$-bit numbers take $O(1)$ time is standard under most algorithmic models.

${ }^{13}$ Because $0 \leq s_{m}^{\prime} \leq t-1$ for all $m \in\{0,1, \ldots, h-1\}$ by line 9 and $\left\{t^{i}\right\}_{i=0}^{h-1}$ has been precomputed, each execution of lines $16-17,19$, and 21 takes $O(t)$ time. Instead, an execution of line 18 takes $O(1)$ time because $\sum_{r=0}^{m-1} s_{r}^{\prime} \cdot t^{r}$ has been precomputed.
} 
Unlike in line 5 of Approx.-Median, Chang's [3] pseudo-distance function requires to query for $d\left(i, i+s t^{k} \bmod n\right)$ for all $i \in\{0,1, \ldots, n-1\}, s \in\{0,1, \ldots, t-$ $1\}$ and $k \in\{0,1, \ldots, h-1\}$ (albeit with a slightly different $t$ ). So his query complexity is $\Theta(h n t)=\Theta\left(h(n) \cdot n^{1+1 / h(n)}\right)$, as compared to our $O\left(n^{1+1 / h(n)}\right)$ in Theorem 9.

\section{Acknowledgments}

The author is supported in part by the Ministry of Science and Technology of Taiwan under grant 106-2221-E-155-013-.

\section{A Proof of Lemma 6}

We begin with the following change-of-variable formula:

Lemma 11 (cf. [3, Lemma 6]). For all $m \in\{0,1, \ldots, h-1\}$ and $s_{m}, s_{m-1}, \ldots$, $s_{0} \in\{0,1, \ldots, t-1\}$,

$$
\begin{aligned}
& \sum_{k=1}^{m} d^{(n-\sigma)}\left(i t^{k}+\sum_{\ell=0}^{k-1} s_{m-\ell} \cdot t^{k-1-\ell}, i t^{k+1}+\sum_{\ell=0}^{k} s_{m-\ell} \cdot t^{k-\ell}\right) \\
= & \sum_{k=0}^{m-1} d^{(n-\sigma)}\left(i t^{k+1}+s_{m} \cdot t^{k}+\sum_{\ell=0}^{k-1} s_{m-1-\ell} \cdot t^{k-1-\ell}, i t^{k+2}+s_{m} \cdot t^{k+1}+\sum_{\ell=0}^{k} s_{m-1-\ell} \cdot t^{k-\ell}\right)
\end{aligned}
$$

Proof. Clearly,

$$
\begin{aligned}
& \sum_{k=1}^{m} d^{(n-\sigma)}\left(i t^{k}+\sum_{\ell=0}^{k-1} s_{m-\ell} \cdot t^{k-1-\ell}, i t^{k+1}+\sum_{\ell=0}^{k} s_{m-\ell} \cdot t^{k-\ell}\right) \\
= & \sum_{k=0}^{m-1} d^{(n-\sigma)}\left(i t^{k+1}+\sum_{\ell=0}^{k} s_{m-\ell} \cdot t^{k-\ell}, i t^{k+2}+\sum_{\ell=0}^{k+1} s_{m-\ell} \cdot t^{k+1-\ell}\right) \\
= & \sum_{k=0}^{m-1} d^{(n-\sigma)}\left(i t^{k+1}+s_{m} \cdot t^{k}+\sum_{\ell=1}^{k} s_{m-\ell} \cdot t^{k-\ell}, i t^{k+2}+s_{m} \cdot t^{k+1}+\sum_{\ell=1}^{k+1} s_{m-\ell} \cdot t^{k+1-\ell}\right) \\
= & \sum_{k=0}^{m-1} d^{(n-\sigma)}\left(i t^{k+1}+s_{m} \cdot t^{k}+\sum_{\ell=0}^{k-1} s_{m-1-\ell} \cdot t^{k-1-\ell}, i t^{k+2}+s_{m} \cdot t^{k+1}+\sum_{\ell=0}^{k} s_{m-1-\ell} \cdot t^{k-\ell}\right),
\end{aligned}
$$

where the first and the last equalities follow from substituting $k$ with $k+1$ and $\ell$ with $\ell+1$, respectively.

Restatement of Lemma 6 (cf. [3, Lemma 6]). For all $i \in\{0,1, \ldots, n-\sigma-1\}$ 
and $m \in\{1,2, \ldots, h-1\}$,

$$
\begin{aligned}
g(i, m) & =t^{m} \sum_{s_{m}=0}^{t-1} d^{(n-\sigma)}\left(i, i t+s_{m}\right) \\
& +\sum_{s_{m}=0}^{t-1} g\left(i t+s_{m} \bmod (n-\sigma), m-1\right) .
\end{aligned}
$$

Proof. By Eq. (33),

$$
\begin{aligned}
& \sum_{s_{m}=0}^{g(i, m)} \sum_{s_{m-1}, s_{m-2}, \ldots, s_{0}=0}^{t-1}\left[d^{(n-\sigma)}\left(i, i t+s_{m}\right)\right. \\
& \left.+\sum_{k=1}^{m} d^{(n-\sigma)}\left(i t^{k}+\sum_{\ell=0}^{k-1} s_{m-\ell} \cdot t^{k-1-\ell}, i t^{k+1}+\sum_{\ell=0}^{k} s_{m-\ell} \cdot t^{k-\ell}\right)\right] \\
= & \sum_{s_{m}=0}^{t-1} t^{m} \cdot d^{(n-\sigma)}\left(i, i t+s_{m}\right) \\
+ & \sum_{s_{m}=0}^{t-1} \sum_{s_{m-1}, s_{m-2}, \ldots, s_{0}=0}^{t-1} \sum_{k=1}^{m} d^{(n-\sigma)}\left(i t^{k}+\sum_{\ell=0}^{k-1} s_{m-\ell} \cdot t^{k-1-\ell}, i t^{k+1}+\sum_{\ell=0}^{k} s_{m-\ell} \cdot t^{k-\ell}\right),
\end{aligned}
$$

where the last equality holds because there are $t^{m}$ tuples $\left(s_{m-1}, s_{m-2}, \ldots, s_{0}\right) \in$ $\{0,1, \ldots, t-1\}^{m}$. Again by Eq. (33),

$$
\begin{aligned}
& g\left(i t+s_{m} \bmod (n-\sigma), m-1\right) \\
= & \sum_{s_{m-1}, s_{m-2}, \ldots, s_{0}=0}^{t-1} \sum_{k=0}^{m-1} d^{(n-\sigma)}\left(\left(i t+s_{m}\right) t^{k}+\sum_{\ell=0}^{k-1} s_{m-1-\ell} \cdot t^{k-1-\ell},\left(i t+s_{m}\right) t^{k+1}+\sum_{\ell=0}^{k} s_{m-1-\ell} \cdot t^{k-\ell}\right)
\end{aligned}
$$

for $s_{m} \in\{0,1, \ldots, t-1\}$, where we use Eq. (5) as well. Eqs. (38)-(39) and Lemma 11 complete the proof.

\section{B Proof of Lemma 7}

For all $m \in\{1,2, \ldots, h-1\}$ and $s_{m}, s_{m-1}, \ldots, s_{0} \in\{0,1, \ldots, t-1\}$, consider whether

$$
\sum_{r=0}^{m} s_{r} \cdot t^{r} \leq \sum_{r=0}^{m} s_{r}^{\prime} \cdot t^{r}
$$

as follows:

(I) If $s_{m}<s_{m}^{\prime}$, then Eq. (40) holds. 
(II) If $s_{m}=s_{m}^{\prime}$, then Eq. (40) holds if and only if $\left(s_{m-1}, s_{m-2}, \ldots, s_{0}\right)$ is the $t$-ary representation of one of $0,1, \ldots, \sum_{r=0}^{m-1} s_{r}^{\prime} \cdot t^{r}$.

(III) If $s_{m}>s_{m}^{\prime}$, then Eq. (40) fails.

Items (I)-(III) follow from the basics of $t$-ary representations.

Lemma 12 (cf. [3, Lemma 7]). For all $m \in\{1,2, \ldots, h-1\}$,

$$
\begin{aligned}
& \sum_{s_{m}=0}^{s_{m}^{\prime}} \sum_{s_{m-1}, s_{m-2}, \ldots, s_{0}=0}^{t-1} \chi\left[\sum_{r=0}^{m} s_{r} \cdot t^{r} \leq \sum_{r=0}^{m} s_{r}^{\prime} \cdot t^{r}\right] \cdot d^{(n-\sigma)}\left(i, i t+s_{m}\right) \\
= & \left(1+\sum_{r=0}^{m-1} s_{r}^{\prime} \cdot t^{r}\right) d^{(n-\sigma)}\left(i, i t+s_{m}^{\prime}\right)+t^{m} \sum_{s_{m}=0}^{s_{m}^{\prime}-1} d^{(n-\sigma)}\left(i, i t+s_{m}\right) .
\end{aligned}
$$

Proof. Items (II) and (I) account for the first and the second terms of the righthand side of Eq. (41), respectively. ${ }^{14}$

The proof of Lemma 7 just uses items (I)-(III) naïvely and with great patience:

Restatement of Lemma 7 (cf. [3, Lemma 7]). For all $i \in\{0,1, \ldots, n-\sigma-1\}$ and $m \in\{1,2, \ldots, h-1\}$,

$$
\begin{aligned}
f(i, m) & =\left(1+\sum_{r=0}^{m-1} s_{r}^{\prime} \cdot t^{r}\right) d^{(n-\sigma)}\left(i, i t+s_{m}^{\prime}\right) \\
& +t^{m} \sum_{s_{m}=0}^{s_{m}^{\prime}-1} d^{(n-\sigma)}\left(i, i t+s_{m}\right) \\
& +f\left(i t+s_{m}^{\prime} \bmod (n-\sigma), m-1\right) \\
& +\sum_{s_{m}=0}^{s_{m}^{\prime}-1} g\left(i t+s_{m} \bmod (n-\sigma), m-1\right) .
\end{aligned}
$$

\footnotetext{
${ }^{14}$ Note that there are $t^{m}$ tuples $\left(s_{m-1}, s_{m-2}, \ldots, s_{0}\right) \in\{0,1, \ldots, t-1\}^{m}$, among which $1+\sum_{r=0}^{m-1} s_{r}^{\prime} \cdot t^{r}$ are $t$-ary representations of one of $0,1, \ldots, \sum_{r=0}^{m-1} s_{r}^{\prime} \cdot t^{r}$.
} 
Proof. We have

$$
\begin{aligned}
& f(i, m) \\
& \stackrel{(32)}{=} \quad \sum_{s_{m}=0}^{t-1} \sum_{s_{m-1}, s_{m-2}, \ldots, s_{0}=0}^{t-1} \chi\left[\sum_{r=0}^{m} s_{r} \cdot t^{r} \leq \sum_{r=0}^{m} s_{r}^{\prime} \cdot t^{r}\right] \cdot\left[d^{(n-\sigma)}\left(i, i t+s_{m}\right)\right. \\
& \left.+\sum_{k=1}^{m} d^{(n-\sigma)}\left(i t^{k}+\sum_{\ell=0}^{k-1} s_{m-\ell} \cdot t^{k-1-\ell}, i t^{k+1}+\sum_{\ell=0}^{k} s_{m-\ell} \cdot t^{k-\ell}\right)\right] \\
& \stackrel{\text { Lemma }}{=} \sum_{s_{m}=0}^{t-1} \sum_{s_{m-1}, s_{m-2}, \ldots, s_{0}=0}^{t-1} \chi\left[\sum_{r=0}^{m} s_{r} \cdot t^{r} \leq \sum_{r=0}^{m} s_{r}^{\prime} \cdot t^{r}\right] \cdot\left[d^{(n-\sigma)}\left(i, i t+s_{m}\right)\right. \\
& \left.+\sum_{k=0}^{m-1} d^{(n-\sigma)}\left(i t^{k+1}+s_{m} \cdot t^{k}+\sum_{\ell=0}^{k-1} s_{m-1-\ell} \cdot t^{k-1-\ell}, i t^{k+2}+s_{m} \cdot t^{k+1}+\sum_{\ell=0}^{k} s_{m-1-\ell} \cdot t^{k-\ell}\right)\right] \\
& \stackrel{\text { item (III) }}{=} \sum_{s_{m}=0}^{s_{m}^{\prime}} \sum_{s_{m-1}, s_{m-2}, \ldots, s_{0}=0}^{t-1} \chi\left[\sum_{r=0}^{m} s_{r} \cdot t^{r} \leq \sum_{r=0}^{m} s_{r}^{\prime} \cdot t^{r}\right] \cdot\left[d^{(n-\sigma)}\left(i, i t+s_{m}\right)\right. \\
& \left.+\sum_{k=0}^{m-1} d^{(n-\sigma)}\left(i t^{k+1}+s_{m} \cdot t^{k}+\sum_{\ell=0}^{k-1} s_{m-1-\ell} \cdot t^{k-1-\ell}, i t^{k+2}+s_{m} \cdot t^{k+1}+\sum_{\ell=0}^{k} s_{m-1-\ell} \cdot t^{k-\ell}\right)\right] .
\end{aligned}
$$

So by Lemma 12 , it remains to prove that

$$
\begin{aligned}
& \sum_{s_{m}=0}^{s_{m}^{\prime}} \sum_{s_{m-1}, s_{m-2}, \ldots, s_{0}=0}^{t-1} \chi\left[\sum_{r=0}^{m} s_{r} \cdot t^{r} \leq \sum_{r=0}^{m} s_{r}^{\prime} \cdot t^{r}\right] \\
& \sum_{k=0}^{m-1} d^{(n-\sigma)}\left(i t^{k+1}+s_{m} \cdot t^{k}+\sum_{\ell=0}^{k-1} s_{m-1-\ell} \cdot t^{k-1-\ell}, i t^{k+2}+s_{m} \cdot t^{k+1}+\sum_{\ell=0}^{k} s_{m-1-\ell} \cdot t^{k-\ell}\right) \\
= & f\left(i t+s_{m}^{\prime} \bmod (n-\sigma), m-1\right)+\sum_{s_{m}=0}^{s_{m}^{\prime}-1} g\left(i t+s_{m} \bmod (n-\sigma), m-1\right) .
\end{aligned}
$$

Separating the left-hand side of Eq. (43) according to whether $s_{m}=s_{m}^{\prime}$ or 


$$
\begin{aligned}
& s_{m} \leq s_{m}^{\prime}-1 \\
& \sum_{s_{m}=0}^{s_{m}^{\prime}} \sum_{s_{m-1}, s_{m-2}, \ldots, s_{0}=0}^{t-1} \chi\left[\sum_{r=0}^{m} s_{r} \cdot t^{r} \leq \sum_{r=0}^{m} s_{r}^{\prime} \cdot t^{r}\right] \\
& \cdot \quad \sum_{k=0}^{m-1} d^{(n-\sigma)}\left(i t^{k+1}+s_{m} \cdot t^{k}+\sum_{\ell=0}^{k-1} s_{m-1-\ell} \cdot t^{k-1-\ell}, i t^{k+2}+s_{m} \cdot t^{k+1}+\sum_{\ell=0}^{k} s_{m-1-\ell} \cdot t^{k-\ell}\right) \\
& =\quad \sum_{s_{m-1}, s_{m-2}, \ldots, s_{0}=0}^{t-1} \chi\left[\sum_{r=0}^{m-1} s_{r} \cdot t^{r} \leq \sum_{r=0}^{m-1} s_{r}^{\prime} \cdot t^{r}\right] \\
& \text {. } \quad \sum_{k=0}^{m-1} d^{(n-\sigma)}\left(i t^{k+1}+s_{m}^{\prime} \cdot t^{k}+\sum_{\ell=0}^{k-1} s_{m-1-\ell} \cdot t^{k-1-\ell}, i t^{k+2}+s_{m}^{\prime} \cdot t^{k+1}+\sum_{\ell=0}^{k} s_{m-1-\ell} \cdot t^{k-\ell}\right) \\
& +\sum_{s_{m}=0}^{s_{m}^{\prime}-1} \sum_{s_{m-1}, s_{m-2}, \ldots, s_{0}=0}^{t-1} \chi\left[\sum_{r=0}^{m} s_{r} \cdot t^{r} \leq \sum_{r=0}^{m} s_{r}^{\prime} \cdot t^{r}\right] \\
& \cdot \quad \sum_{k=0}^{m-1} d^{(n-\sigma)}\left(i t^{k+1}+s_{m} \cdot t^{k}+\sum_{\ell=0}^{k-1} s_{m-1-\ell} \cdot t^{k-1-\ell}, i t^{k+2}+s_{m} \cdot t^{k+1}+\sum_{\ell=0}^{k} s_{m-1-\ell} \cdot t^{k-\ell}\right) \\
& \stackrel{\text { item }}{=} \sum_{s_{m-1}, s_{m-2}, \ldots, s_{0}=0}^{t-1} \chi\left[\sum_{r=0}^{m-1} s_{r} \cdot t^{r} \leq \sum_{r=0}^{m-1} s_{r}^{\prime} \cdot t^{r}\right] \\
& \cdot \quad \sum_{k=0}^{m-1} d^{(n-\sigma)}\left(i t^{k+1}+s_{m}^{\prime} \cdot t^{k}+\sum_{\ell=0}^{k-1} s_{m-1-\ell} \cdot t^{k-1-\ell}, i t^{k+2}+s_{m}^{\prime} \cdot t^{k+1}+\sum_{\ell=0}^{k} s_{m-1-\ell} \cdot t^{k-\ell}\right) \\
& +\sum_{s_{m}=0}^{s_{m}^{\prime}-1} \sum_{s_{m-1}, s_{m-2}, \ldots, s_{0}=0}^{t-1} \\
& \sum_{k=0}^{m-1} d^{(n-\sigma)}\left(i t^{k+1}+s_{m} \cdot t^{k}+\sum_{\ell=0}^{k-1} s_{m-1-\ell} \cdot t^{k-1-\ell}, i t^{k+2}+s_{m} \cdot t^{k+1}+\sum_{\ell=0}^{k} s_{m-1-\ell} \cdot t^{k-\ell}\right) \\
& \stackrel{(5)}{=} \quad \sum_{s_{m-1}, s_{m-2}, \ldots, s_{0}=0}^{t-1} \chi\left[\sum_{r=0}^{m-1} s_{r} \cdot t^{r} \leq \sum_{r=0}^{m-1} s_{r}^{\prime} \cdot t^{r}\right] \\
& \quad \sum_{k=0}^{m-1} d^{(n-\sigma)}\left(\left(i t+s_{m}^{\prime} \bmod (n-\sigma)\right) t^{k}+\sum_{\ell=0}^{k-1} s_{m-1-\ell} \cdot t^{k-1-\ell},\right. \\
& \left.\left(i t+s_{m}^{\prime} \bmod (n-\sigma)\right) t^{k+1}+\sum_{\ell=0}^{k} s_{m-1-\ell} \cdot t^{k-\ell}\right) \\
& +\sum_{s_{m}=0}^{s_{m}^{\prime}-1} \sum_{s_{m-1}, s_{m-2}, \ldots, s_{0}=0}^{t-1} \\
& \sum_{k=0}^{m-1} d^{(n-\sigma)}\left(\left(i t+s_{m} \bmod (n-\sigma)\right) t^{k}+\sum_{\ell=0}^{k-1} s_{m-1-\ell} \cdot t^{k-1-\ell}\right. \\
& \left.\left(i t+s_{m} \bmod (n-\sigma)\right) t^{k+1}+\sum_{\ell=0}^{k} s_{m-1-\ell} \cdot t^{k-\ell}\right) \\
& \stackrel{(32)-(33)}{=} f\left(i t+s_{m}^{\prime} \bmod (n-\sigma), m-1\right)+\sum_{s_{m}=0}^{s_{m}^{\prime}-1} g\left(i t+s_{m} \bmod (n-\sigma), m-1\right) \text {. }
\end{aligned}
$$




\section{References}

[1] M. Agrawal, N. Kayal, and N. Saxena. PRIMES is in P. Annals of Mathematics, 160(2):781-793, 2004.

[2] P. Bose, A. Maheshwari, and P. Morin. Fast approximations for sums of distances, clustering and the Fermat-Weber problem. Computational Geometry, 24(3):135-146, 2003.

[3] C.-L. Chang. A deterministic sublinear-time nonadaptive algorithm for metric 1-median selection. Theoretical Computer Science, 602:149-157, 2015.

[4] C.-L. Chang. A note on 1-median selection in metric spaces. In Proceedings of the 32nd Workshop on Combinatorial Mathematics and Computation Theory, pages 51-54, Taichung, Taiwan, 2015. This workshop does not publish proceedings.

[5] C.-L. Chang. Metric 1-median selection: Query complexity vs. approximation ratio. ACM Transactions on Computation Theory, 9(4):20:1-20:23, 2018.

[6] K. Chen. On coresets for $k$-median and $k$-means clustering in metric and Euclidean spaces and their applications. SIAM Journal on Computing, 39(3):923-947, 2009.

[7] T. H. Cormen, C. E. Leiserson, R. L. Rivest, and C. Stein. Introduction to Algorithms. The MIT Press, 3rd edition, 2009.

[8] S. Guha, A. Meyerson, N. Mishra, R. Motwani, and L. O'Callaghan. Clustering data streams: Theory and practice. IEEE Transactions on Knowledge and Data Engineering, 15(3):515-528, 2003.

[9] P. Indyk. Sublinear time algorithms for metric space problems. In Proceedings of the 31st Annual ACM Symposium on Theory of Computing (STOC'99), pages 428-434, 1999.

[10] P. Indyk. High-dimensional computational geometry. PhD thesis, Stanford University, 2000.

[11] V. Kabanets and R. Impagliazzo. Derandomizing polynomial identity tests means proving circuit lower bounds. Computational Complexity, 13(1-2):146, 2004. 
[12] C. Kaklamanis, D. Krizanc, and T. Tsantilas. Tight bounds for oblivious routing in the hypercube. Mathematical Systems Theory, 24(1):223-232, 1991.

[13] A. Kumar, Y. Sabharwal, and S. Sen. Linear-time approximation schemes for clustering problems in any dimensions. Journal of the ACM, 57(2):5, 2010 .

[14] L. G. Valiant. A scheme for fast parallel communication. SIAM Journal on Computing, 11(2):350-361, 1982.

[15] S. Wasserman and K. Faust. Social Network Analysis: Methods and Applications. Cambridge University Press, 1994.

[16] B.-Y. Wu. On approximating metric 1-median in sublinear time. Information Processing Letters, 114(4):163-166, 2014. 